\title{
DU RÉTRO AU NÉO, ENTRE NOSTALGIE ET RÉINVENTION. DISCOURS, OBJETS, USAGES DANS LES CULTURES MÉDIATIQUES CONTEMPORAINES
}

\author{
NOTE INTRODUCTIVE
}

Sébastien Fevry ${ }^{1}$, Sarah Sepulchre ${ }^{2}$ et Marie Vanoost ${ }^{3}$

En décembre 2016 se tenait dans le lieu particulièrement propice du Mundaneum ${ }^{4}$ à Mons un colloque international organisé par le GIRCAM ${ }^{5}$ consacré aux tendances rétro et néo dans nos cultures contemporaines. Ce colloque était motivé par la volonté d'interroger la récurrence et la signification de phénomènes culturels et médiatiques qui jusqu'alors avaient été peu étudiés par la recherche francophone ${ }^{6}$, du moins dans le périmètre des études culturelles et des sciences de l'information et de la communication ${ }^{7}$. Notre attention portait sur un ensemble

1 Sébastien Fevry est professeur à l'Université catholique de Louvain et coordinateur du GIRCAM.

2 Sarah Sepulchre est professeure à l'Université catholique de Louvain et coordinatrice du GIRCAM.

3 Marie Vanoost est chargée de recherches du F.R.S.-FNRS à l'Université catholique de Louvain au sein de l'Observatoire de Recherche sur les Médias et le journalisme (ORM).

4 http://www.mundaneum.org/

5 Groupe Interdisciplinaire de Recherche sur les Cultures et les Arts en Mouvement.

6 Notons la parution récente d'un numéro spécial de la revue Le temps des médias consacré à l'Âge d'or (Fantin, Le Hégarat, 2016).

7 Pour une présentation synthétique des recherches actuellement menées sur la nostalgie, on se rapportera à l'article de Katharina Niemeyer « Du mal du pays aux nostalgies numériques. Réflexions sur les liens entre nostalgie, nouvelles technologies et médias » placé en début de ce numéro.

Recherches en communication, $n^{\circ} 46$ - Article publié le 24/04/2018 
de modes, de mots et d'usages qui tous signalaient un retour du passé au sein du présent et l'emprise d'une vague nostalgique pouvant se décliner en une grande variété d'objets et de processus d'appropriation : retour des vinyles et des cassettes audio, mode vintage, films rétro, vague sépia, actions de recyclage et de customisation, productions rétro-futuristes...

Cependant, la question posée à l'entrée du colloque n'engageait pas seulement à constater la diversité de ces phénomènes, mais à prendre en compte le caractère profondément ambigu de tendances nostalgiques qui sont à la fois tournées vers le passé et ouvertes sur l'avenir, liées à des désirs régressifs et à une volonté conservatrice, mais également porteuses de réinvention et de subversion. C'est pourquoi il fallait prendre soin de ne pas séparer les objets et discours nostalgiques de leurs usages pour les intégrer dans des dynamiques socio-culturelles où se croisent ancrage dans le passé, attention au temps présent et regard orienté vers le futur.

Un autre impératif du colloque était de ne pas s'en tenir à des considérations générales, mais de privilégier des objets emblématiques des modes rétro, le concept d'objet étant entendu de façon large en articulant les différentes fonctions que Bruno Latour (2007) accorde aux objets peuplant notre quotidien, à savoir la dimension d'outil et d'écran, mais aussi la fonction d'échange et de médiation. Par 'objet', on pouvait donc entendre une série, un jeu vidéo, un film, une bande dessinée, une publicité, un dispositif comme le diorama ou un support médiatique comme les mooks, sans oublier des objets plus 'traditionnels', certains manipulables et de taille domestique comme le tourne-disque, la cassette audio ou la carte de hockey, d'autres habitables et de plus grande taille comme la péniche ou le zeppelin. Bref, le colloque avait l'ambition de dresser un inventaire critique et réflexif des différents objets où se (ré)investissent des rapports multiples et différenciés au passé.

Ce colloque organisé en 2016 constitue le point de départ du présent numéro. En plus de certaines communications dorénavant transformées en articles, s'ajoutent d'autres interventions qui ont fait suite à l'appel à contribution lancé par la revue Recherches 
en Communication. Au total, ce dossier comptera plus d'une quinzaine de textes, ce qui témoigne bien de l'intérêt des chercheurs et chercheuses pour les questions liées aux différentes modalités de la nostalgie dans nos cultures médiatiques. Par la richesse de son contenu et la diversité de ces interventions, cette publication rend visible l'ambition énoncée précédemment, à savoir livrer un panorama critique, mais forcément inachevé, des différentes modalités de la nostalgie médiatique en ce début du $21^{\mathrm{e}}$ siècle.

Par rapport aux axes directeurs du colloque, le numéro permet de préciser certaines lignes de force, à commencer par le fait que le rapport au passé n'est jamais cultivé de manière passive, mais qu'il témoigne toujours d'une réappropriation active de la part des spectateurs ou des usagers. Ainsi, les séries télévisées participent à construire une 'mémoire culturelle' et deviennent des objets transactionnels pour le public, comme le défend l'article sur la série Life on Mars qui examine le procédé du voyage dans le temps. Toujours sur l'axe de la temporalité, les textes rassemblés ici confirment que l'étude des nostalgies médiatiques consiste à prêter attention à l'entrecroisement des temps, à la façon dont passé, présent et futur cohabitent parfois dans un même objet ou dans une même représentation, ainsi que le montrent l'étude sur le diorama et celle sur le zeppelin et le rétro-futurisme.

Une autre ligne de force apparaissant au fil des pages tient dans la capacité qu'a la nostalgie d'interroger les rapports entre cultures savante et populaire, art et divertissement, puisque le passage du temps peut faire entrer des productions autrefois jugées comme populaires dans le champ de l'art contemporain (voir l'article sur le diorama). Plus largement, on peut aussi estimer - ainsi qu'en témoignent les articles sur les comic strips américains, les canards en plastique ou les cartes de hockey -, que la nostalgie, pour être pleinement appréciée, tend à réclamer sa propre expertise, à développer « un rapport cultivé à la culture non cultivée » (Donnat citée par Peyron, 2008, p. 355) et à susciter certaines pratiques réflexives, ainsi que le souligne notamment le texte portant sur les jeux d'autoréférence dans la publicité contemporaine. 
Enfin, dernière ligne de force, si l'on se tient davantage sur un axe médiatique, on s'aperçoit que la nostalgie porte tout autant sur le média que sur le contenu véhiculé par celui-ci, ainsi que l'illustrent exemplairement les analyses sur le tourne-disque, les mooks ou la cassette audio, pour ne prendre que quelques exemples parmi d'autres. Dépassant les oppositions tranchées entre hors-ligne et en ligne, ces technonostalgies (van der Heijden, 2015) ont aussi bien trait aux médias analogiques que numériques, comme le rappelle utilement Katharina Niemeyer en ouverture de ce numéro.

À la fois sentiment et processus qui tendrait à nostalgiser (Niemeyer, 2014) certains objets ou discours, la nostalgie apparaît également dans ce dossier comme une démarche de questionnement, le point d'entrée d'une réflexion dont le principal effet retour consiste finalement à revisiter nos mémoires médiatiques, à interroger les traces et les objets qui les constituent, cela dans des logiques temporelles complexes, situées entre passé, présent et futur.

\section{Références}

Fantin, E. \& Le Hégarat, Th. (éd.) (2016). « L'Âge d'or », Le Temps des médias, 27/2. Heijden, van der, T. (2015). Technostalgia of the Present: From Technologies of Memory to a Memory of Technologies. European Journal of Media Studies, 4 (2), 103-121.

Latour, B. (2007). Une sociologie sans objet? Remarques sur l'interobjectivité. Dans O. Debary \& L. Turgeon, Objets et Mémoires. Paris-Québec : Fondation Maison des sciences de l'homme/Les Presses de l'Université Laval, 37-57.

Niemeyer, K. (Ed.) (2014). Media and Nostalgia. Yearning for the past, present and future. Basingstoke : Palgrave Macmillan.

Peyron, D. (2008). Quand les œuvres deviennent des mondes. Une réflexion sur la culture de genre contemporaine à partir du concept de convergence culturelle. Réseaux, 2, n 148-149, 335-368.

\section{(c) $(1) \Theta \Theta$}

Publié sous la licence Creative Commons

«Attribution - Pas d'Utilisation Commerciale - Pas de Modification 4.0 International» (CC BY-NC-ND) 